\title{
Intelligent Management System of Power Network Information Collection Under Big Data Storage
}

\author{
Yingying Qin ${ }^{1 . *}$, Hua Xuan ${ }^{1}$ and Bailin Zhang $^{2}$ \\ ${ }^{1}$ School of Management Engineering, Zhengzhou University, Zhengzhou 450001, China \\ ${ }^{2}$ Inspur Electronic Information Industry Co. ,Ltd, Jinan 250101, China \\ Corresponding Email: 1484261055@qq.com
}

\begin{abstract}
With the development of economy and society, big data storage in enterprise management has become a problem that can't be ignored. How to manage and optimize the allocation of tasks better is an important factor in the sustainable development of an enterprise. Now the enterprise information intelligent management has become a hot spot of management mode and concept in the information age. It presents information to the business managers in a more efficient, lower cost, and global form. The system uses the SG-UAP development tools, which is based on Eclipse development environment, and suits for Windows operating system, with Oracle as database development platform, Tomcat network information service for application server. The system uses SOA service-oriented architecture, provides RESTful style service, and HTTP(S) as the communication protocol, and JSON as the data format. The system is divided into two parts, the front-end and the backs-end, achieved functions like user login, registration, password retrieving, enterprise internal personnel information management and internal data display and other functions.
\end{abstract}

\section{Introduction}

The demand of acquisition system is increasing, it is imperative to improve the data processing performance of the electric information acquisition system through leading technology. The amount of data generated by electricity is considerable, Therefore the data storage and mining analysis has become the most important one, including the selection of database and optimization of the SQL statement. These are precisely the direction of this research.

For the implementation plan of Henan electric power company "three years to enhance the quality of rural power", to support the carry out of Henan county company management, to deepen the application of measurement acquisition, and measurement acquisition related business city, county integration and other work, based on the principle of the establishment of the main station expansion and function upgrading of the electric information acquisition system issued by the state power grid, designed and realized the management system of Henan province electric information (including the County Department and the equipment, etc.). The upper controllers improved their work effectively, they can observe the power dynamics in real time, which is of practical significance.

Intelligent enterprise information management has become the main model in the information age, which is changing the way managers works. Firstly, the management of big data in enterprises has become a difficult problem in modern times, while using information management can find the required information more quickly, and improve work efficiency greatly. Secondly, which can make the management of enterprise data information organized, facilitate to operation the company. Thirdly, as diversified and personalized management is becoming more and more common in our daily fast pace of life, management software which is highly efficient and easy to operate is more popular.

Big data storage problem has been discussed by many scholars in related fields, and has achieved a lot of research results so far. For example, Literature [1] based on the analysis of cloud computing platform, a uniform hash storage algorithm was proposed for big data storage and fast access problem. Literature [2] introduced the big data management under the new storage technology. The information management system of power network was described in literature [3-5], among which, Literature [3] introduced smart grid information management system under the multi-agent technology. Literature [4] analyzed the complexity of smart grid information management system. Literature [5] studied the information share and control management under the smart grid information management system. Literature [6] introduced the features and functions of SQL Server database. Literature [7] introduced the system evaluation from traditional database to emergent big data. For a large number of enterprise data management issues, literature [8] studied the business intelligence data management. Literature [9] used J2EE, Oracle 9i design applications and upload to cloud under the enterprise ERP management system of the cloud computing platform. Based on the above research, this paper designed and developed power grid information management system to solve the effective management of big data, information diversity problems and so on. 
The paper is organized as follows. The next section, section 2 introduces the technique used in this system. In section 3 , we show the architecture design in detail. In section 4, we introduce the core modules, especially it's implementation.Finally,some conclusion are made in section 5.

\section{Technical description}

The development platform of this system is: operating system Microsoft Windows; programming language Java/Ajax; development tool UAP Studio 2.0.0; Database Oracle 10g; Java application server Tomcat 6.0, IDE Eclipse as the core of the enterprise software, to combine database, development programs and application servers quickly. The development owns a variety of languages, complete coding, debugging, testing and publishing, supports HTML, JavaScript, Sql, Spring, Hibernate, CSS, Ajax, JSON, jQuery technologies and so on. The platform uses two level technical service system, and the technical service includes technical consulting, training and examination, assistant design, design review, development direction, operation, maintenance support and so on. In the UAP, the Rest styles are interactive with the front and the back ends, Written in the URL address in the foreground form or the underlying code and the background to interact to realize system front desk and the background management individually.

The server framework as shown in Figure 1, the application system based on OSGI was embedded in the framework of application server. With the advantages of Web applications and advanced dynamic, modular characteristics, realized the high coupling between the application system modules. The platform was composed of a modular framework, which is the smallest unit that can run on the platform. Micro kernel inherited the thought and technology of SoTower platform, using Servlet bridge mode, the OSGI framework is embedded in the application container to run. The internal application of the platform and all the functions of the business were assigned to specific modules, which runs in the OSGI framework.

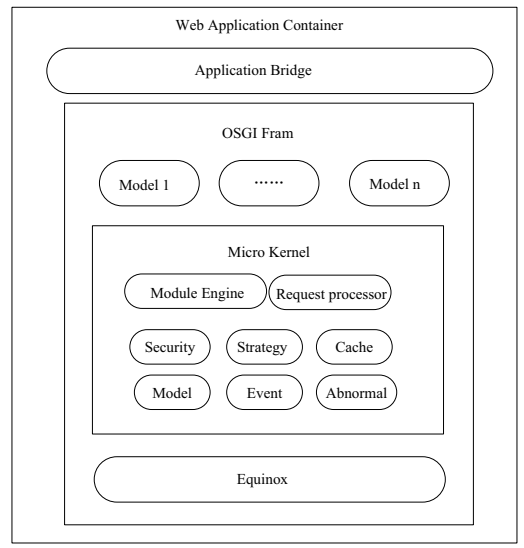

Fig.1. Server framework.

The design discarded the disadvantages of the previous logic module partition-cross store each function module file: business modules are easy to interfere with each other, reduce the efficiency of the development of business applications, which also has a certain influence on the stability of the product, SG-UAP platform micro kernel solved these problems.

The server was divided into three layers, control layer, logic layer and persistence layer. Control layer, also called interaction layer, the service interaction section is one of the most important parts of the core framework, which is responsible for receiving and processing requests from browser or system, providing users data transmission format, packet sending, URL handling of the distribution and other functions. Logic layer is generally considered as the service layer of SG-UAP project hierarchy, between with the interaction layer and the persistence layer, which generally dependent on the persistence layer not the interaction layer. The responsibility of the logic layer is to encapsulate the business logic. In SG-UAP, logic layer technology mainly refers to logic component, to a certain extent, simplified the use of the business development, web services, AOP and other functions. The use of the persistence layer is mainly reflected in the resource allocation and the call of the persistence method. By default, the platform configures a SessionFactory object and Hibernate file path in the file for the user automatically. Hibernate can perfectly be used on the platform, be good division of application levels, conduct transaction management and data access independently, improve the degree of polymerization between the modules greatly and reduce the coupling degree of the application objects.

ASP, AJAX, Oracle database, Annotation technology and Hibernate Technology were used in this system, AJAX technology integrated the development of CS, which can solve the problem more effectively. The most widely used database is Oracle and MySQL, Oracle suits large and medium-sized enterprises, while MySQL suits small and medium enterprises. As a large number of data requires the performance of the database very high, small database is difficult to meet the needs, so Oracle was chosen. 


\section{Architecture design}

\subsection{Functional analysis}

The system platform uses SOA framework, SG-UAP software combined with Oracle database to achieve the general management operation of enterprise information, which contains the following function modules:

(1) User registration: the system needs to be registered for the first time, and needs to verify identity. The users are unique, the identity card number and the nickname of the registered person are unique.

(2) Retrieve password: Once the password error, users will not be able to enter the system, users needs to retrieve the password, and the nickname, ID number and other information is essential, must be consistent with the database data, otherwise the password will not be found.

(3) Validate logon: User login needs input name and password, considering the same name, so does not use the real name as the logon condition, and nickname as the login name, which requires the nickname must be unique. Click on the login button to verify the information.

(4) Department equipment information management: through a form, the department equipment information will be displayed to the manager dynamically. Managers query the associated information through the index, number and name. Different forms can be chosen to display the department equipment information, giving the users visual enjoyment.

(5) Staff information management: the information of staff is displayed on the left side of the page, through click the staff information node then display the corresponding information, which is convenient for the manager to manage.

(6) Enterprise basic information management: the enterprise information displays the related registration information preview to the users. Through this module, users can use histogram to view all of the registered capital of the enterprise and security authentication information simply.

(7) Qualification information management of state grid electric power enterprise: to record the information of enterprise registration certificate in the form of DataGrid.

(8) Security license information: to browse the safety license information of enterprises in the form of Grid Complex.

\subsection{System structure diagram}

The following figure 2 shows the design ideas of the state grid information acquisition system module, including the front-end and the back-end. The back-end module contains four parts: the persistent layer, the control layer, the logic layer and the UI layer. The first three sections are needed to interact with the database.

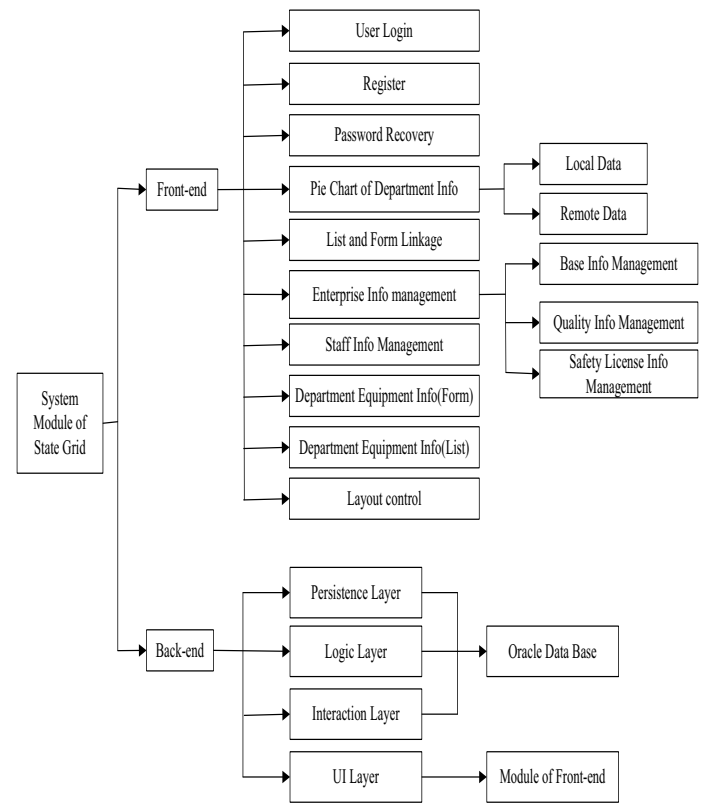

Fig.2. The diagram of system structure.

\subsection{System use case diagram}

The relationship between all modules of the system and the user is presented in the form of a use case diagram 3. Users can add, delete, edit and search the department information, equipment information, employee information and the State 
Grid Electric Power Enterprise Information. The system provided corresponding interfaces for different users. If you do not have the authority to $\log$ in the system, you need to register the account. If you forget the password, you can apply for resetting the password. If you have an account and the authentication information is correct, you can re verify and login.

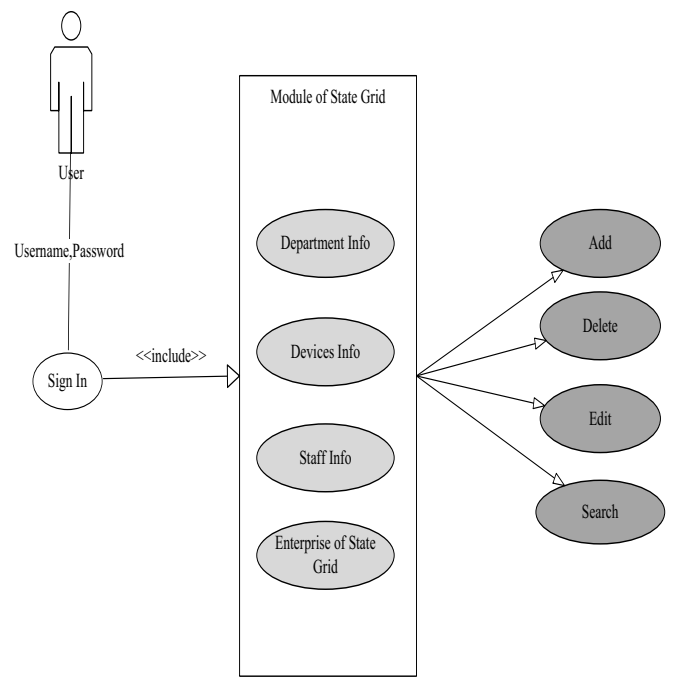

Fig.3. The diagram of system use case.

\subsection{ER chart}

The establishment of the primary key and the dependency relationship between the various data tables facilitates the index query, which can optimize the operation of the database. The relationship between tables is established by primary keys and dependencies, and the tables satisfy the criteria for implementing referential integrity, avoiding some incorrect operations, which can manage the data table effectively. The integral ER chart is shown as Figure 4.

\section{Implementation of core modules}

When users enter the homepage, they can manage enterprise information, for example, add / delete / edit / query / add attachments and other functions can be achieved, changing the previous operating location, the new, edit, delete and other operation can be integrated into each record, which can improve work efficiency.

Page layout as shown in Figure 5, HSplit as the main interface, dividing the page into two parts, displaying the basic information DataGrid in HSplit(0). In addition, creating a VSplit in HSplit(1), which divided the page into two parts vertically display, and VSplit(0) used to store chart information, setting the enterprise name and registered capital for the vertical and horizonta coordinates, the histogram shows the data information is stored in VSplit(1), the property can be set to display data in various forms.

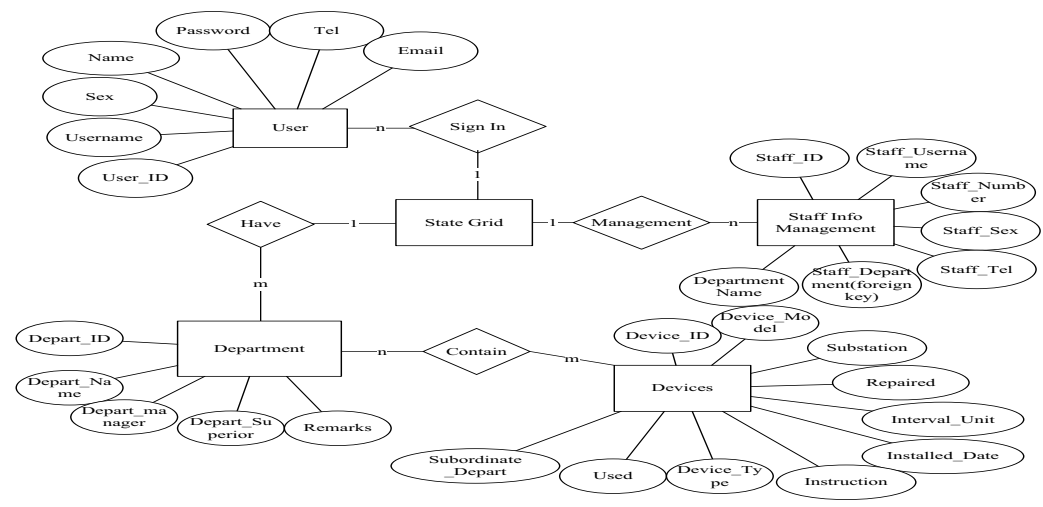

Fig.4. The integral ER chart of electric power information management. 


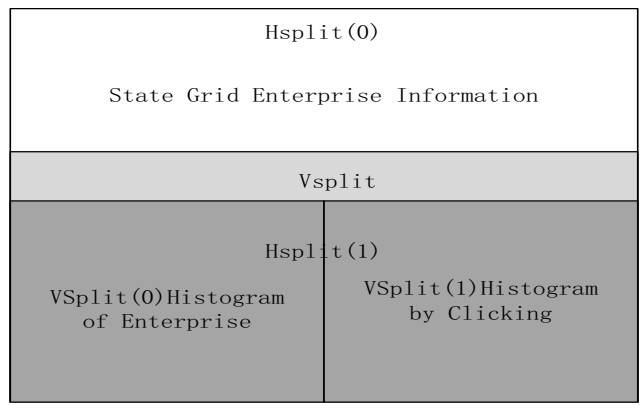

Fig. 5. The diagram of page layout.

\section{Conclusion}

In order to change the traditional enterprise management model, this paper provides a novel management plan, using software to manage the large enterprise data information, which can improve the efficiency of enterprise management greatly and save costs. For example, the existing power information acquisition system storage capacity, the data will not be updated in real time, so the operation time, reaction efficiency and access time should be improved. The use in the SQL statement is optimized to better save access to query time, do a great improvement of big data storage and access. This paper studied the development and implementation of this system, both in the visual and the operation gives users a new experience, which is simple and easy to operate. Further research may consider designing and managing more complex database, and web page dynamic display, more complex needs analysis and other factors.

\section{References}

1. SONG Y Q,ZHOU G L,ZHU Y L,LI L,WANG L W,WANG D W. Storage optimization and parallel processing of condition monitoring big data of transmission and transforming equipment based on cloud platform. Proceedings of the CSEE, ,35,255-267, (2015).

2. JIN P Q,HAO X J,YUE L H.A survey on storage architectures and core algorithms for big data management on new storages.Computer Engineering \& Science,35,12-24,(2013).

3. LIU X M,TIAN H Y,QIN C. Research on smart grid information management system based on complex scientific management thinking and multi agent system technology. Power System Technology, 36,204-208,(2012).

4. LIU X M,JU J. Analysis of the complexity features for information management system of smart grid. Journal of North China Electric Power University (Social Sciences), 2, 20-24, (2013).

5. CHENG S J, LI X Y,ZHANG Z Z. Entire-grid-area information-sharing and integrated applications in united information system for smart grid. Proceedings of the CSEE, 31, 8-31, (2011).

6. YAN X. Research of the characteristics and basic functions of the SQL server database. Value Engineering, 31,229-231, (2012).

7. JIN C Q,QIAN W N,ZHOU M Q,ZHOU AO Y. Benchmarking data management systems: from traditional database to emergent big data. Chinese Journal of Computers, 38,18-34, (2015).

8. SHEN Z P,XIE Y Q. Business Intelligence data management based on corporate information factory. Information Science, 31,102-106, (2013).

9. ZHAO L. Design and implementation of enterprise ERP system based on cloud computing platform. Electronic Design Engineering,24 ,47-49, (2016). 